\title{
Layered double hydroxide as carrier of herbicide, 2-methyl-4-chlorophenoxy acetic acid : physicochemical characterization and controlled release properties
}

\begin{abstract}
A new organic-inorganic hybrid nanocomposite Zn-Al-MCPA layered double hydroxide was prepared by intercalation of 2-methyl-4-chlorophenoxy acetic acid (MCPA), into Zn-Allayered double hydroxide (ZAL) at various concentration of MCPA ranging from 0.1 to 0.7 . The $\mathrm{pH}$ of the formulation was kept constant at 7.5. Well-ordered hybrid nanocomposite was obtained with $0.4 \mathrm{M}$ MCPA with an expansion of basal spacing from $8.9 \AA$ in the ZAL $\mathrm{s}$ resemblance peaks of to $19.7 \AA$ in the resulting nanocomposite. The FTIR spectra of the nanocomposite shows resemblance peaks of the MCPA and Zn-Al-layered double hydroxide indicates the inclusion of MCPA into the layered double hydroxide with loading of MCPA found to be $45 \%$ (w/w). The release of MCPA into various aqueous solution was found to be dependent on the anion in the aqueous solution, in the order of phosphate $>$ sulphate $>$ nitrate with the percentage release of $80 \%, 44 \%$ and $8 \%$, respectively. This study shows that $\mathrm{Zn}-\mathrm{Al}-$ layered double hydroxide can be used as a host carrier for herbicide, MCPA with controlled release capability.
\end{abstract}

Keyword: 2-methyl-4-chlorophenoxy acetic acid; Herbicides; Hybrid nanocomposite; Layered double hydroxide. 